\title{
FABER POLYNOMIALS AND SPECTRUM LOCALISATION
}

\author{
OSCAR DEVYS
}

\begin{abstract}
Let $K$ be a compact connected subset of the complex plane, of non-void interior, and whose complement in the extended complex plane is connected. Denote by $F_{n}$ the $n$-th Faber polynomial associated with $K$. The aim of this paper is to find suitable Banach spaces of complex sequences, $\mathcal{R}$, such that statements of the following type hold true: if $T$ is a bounded linear operator acting on the Banach space $\mathcal{X}$ such that $\left(\left\langle F_{n}(T) x, x^{*}\right\rangle\right)_{n \geq 0} \in \mathcal{R}$ for each pair $\left(x, x^{*}\right) \in \mathcal{X} \times \mathcal{X}^{*}$, then the spectrum of $T$ is included in the interior of $K$. Generalisations of some results due to W. Mlak, N. Nikolski and J. van Neerven are thus obtained and several illustrating examples are given. An interesting feature of these generalisations is the influence of the geometry of $K$ and the regularity of its boundary.
\end{abstract}

\section{INTRODUCTION}

Faber polynomials are now classical objects of study in complex analysis, function theory and approximation theory. We use in this paper Faber polynomials as a basic tool to study the following "spectrum localisation problem" in operator theory: identify conditions under which the spectrum of a Banach space bounded linear operator is included in the interior of a given compact of the complex plane. Other instances of the use of Faber polynomials for various problems in operator theory are $[1,2,3,4,17]$.

Computing or estimating the spectrum of a large matrix, or, more generally, of a bounded linear operator acting on a complex Banach space is an important problem in spectral theory, with possible applications in numerical analysis (see for instance [12]). The classical spectral radius formula ([5])

$$
r(T)=\lim _{n \rightarrow \infty}\left\|T^{n}\right\|^{1 / n},
$$

going back to Arne Beurling and Israel Gelfand, allows to find or estimate the radius $r(T)=\sup \{|\lambda|: \lambda \in \sigma(T)\}$ of the smallest closed disc in the plane centered in the origin and containing the spectrum $\sigma(T)$ of the given operator $T$. The condition $r(T)<1$, corresponding to the exponential stability of $T$, is an important one for applications. In order to better localise the spectrum and to find inclusions of the form $\sigma(T) \subset K$ or $\sigma(T) \subset \operatorname{int}(K)$ for some simply connected compact $K$ in the complex plane, the right tool to consider is the asymptotic behaviour of the sequence $\left(F_{n}(T)\right)_{n>0}$, where $F_{n}$ is the Faber polynomial of degree $n$ associated with $K$. We are interested to generalise in this vein (replacing the closed unit disc by more general compacts) several existing results in the literature $[11,19,13,14,18]$. An interesting feature of our study is the influence 
of the geometry of the compact and the regularity of its boundary on the results obtained about the localisation of the spectrum.

The present paper is organized as follows. Section 2 is dedicated to the definition and basic properties of Faber polynomials. We present the influence of the regularity of the boundary of the compact on the behaviour of these polynomials and present known applications in operator theory. In Sections 3 and 4 we present two approaches leading to different criteria for the spectrum to be included in the interior of a compact. Some generalisations of results of Mlak ([11]), Weiss ([19]), van Neerven $([18])$ and Nikolski $([13,14])$ are given there. We give in Section 5 some explicit estimates based upon the preceding criteria.

\section{Preliminaries on FABer POLYNOMials}

2.1. Definition and examples. We present here some classical results about Faber polynomials which will be used later on. We follow the setting of [16]. The reader is referred to [16] or [8] for more details about Faber polynomials and related topics.

Let $K$ be a compact connected subset of the complex plane $\mathbb{C}$, different from a singleton, and whose complement is connected. From the Riemann mapping theorem, we know that there exists a unique conformal map $\psi: \overline{\mathbb{D}}^{c} \longrightarrow K^{c}$ such that

$$
\psi(\infty)=\infty \quad \text { and } \quad \psi^{\prime}(\infty)>0 .
$$

The map $\psi$ has a Laurent expansion for $|w|>1$ of the form

$$
\psi(w)=\beta w+\beta_{0}+\beta_{1} w^{-1}+\cdots+\beta_{k} w^{-k}+\ldots
$$

where $\beta>0$ is the transfinite diameter, or (logarithmic) capacity, of $K$.

Let $\phi$ be the inverse function of $\psi$. The map $\phi: K^{c} \longrightarrow \overline{\mathbb{D}}^{c}$ has a Laurent expansion in a neighborhood of infinity of the form

$$
\phi(z)=\frac{1}{\beta} z+b_{0}+b_{1} z^{-1}+\cdots+b_{k} z^{-k}+\ldots
$$

For $n \in \mathbb{N}$, the polynomial part of the Laurent expansion of $\phi(z)^{n}$ is called the Faber polynomial of order $n$ and is denoted by $F_{n}$. The Faber polynomial of order $n$ has degree $n$ and leading coefficient $1 / \beta^{n}$. We consider the function $\omega_{n}$ defined by the following equation

$$
\phi(z)^{n}=F_{n}(z)+\omega_{n}(z), \quad\left(z \in K^{c}\right) .
$$

Then $z \mapsto \omega_{n}(z)$ is an analytic bounded function on $K^{c}$ which tends to 0 at infinity.

For any $R>1$, let $\Gamma_{R}$ be the analytic Jordan curve $\{\psi(w):|w|=R\}$. Denote by $G_{R}$ its interior. If $z$ is in $G_{R}$ then we have

$$
F_{n}(z)=\frac{1}{2 i \pi} \int_{\Gamma_{R}} \frac{\phi(\zeta)^{n}}{\zeta-z} d \zeta=\frac{1}{2 i \pi} \int_{|w|=R} \frac{w^{n} \psi^{\prime}(w)}{\psi(w)-z} d w
$$


One can deduce from this relation that the Faber polynomials satisfy the following asymptotic relations

$$
\lim _{n \rightarrow \infty}\left|F_{n}(z)\right|^{1 / n}=|\phi(z)| \text { for all } z \notin K \text { and } \lim _{n \rightarrow \infty}\left|F_{n}(z)\right|^{1 / n} \leq 1 \text { for all } z \in K .
$$

It also implies that for any fixed $z$ in $G_{R},\left(F_{n}(z)\right)_{n \geq 0}$ is the sequence of the Laurent coefficients of the map

$$
w \mapsto \frac{\psi^{\prime}(w)}{\psi(w)-z}, \quad|w|>R,
$$

in the neighborhood of the point $w=\infty$. Therefore the generating function of the Faber polynomials is given by

$$
\frac{\psi^{\prime}(w)}{\psi(w)-z}=\sum_{n=0}^{\infty} \frac{F_{n}(z)}{w^{n+1}}, \quad\left(|w|>R, z \in G_{R}\right) .
$$

The Faber polynomial of order $n$ of the disk centered at $z_{0}$ and of radius $R$ is given by the formula $F_{n}(z)=\left(\left(z-z_{0}\right) / R\right)^{n}$. The series of Faber polynomials of an analytic function in the neighborhood of the compact $K$ is a generalisation of the Taylor expansion of an analytic function in an open disc. More precisely, we have the following theorem (cf. [16, Theorem 3.2.2]).

Theorem 2.1 ([16]). An analytic function $f$ in a neighborhood of the compact $K$ can be uniquely expanded in a series of Faber polynomials with uniform convergence on a neigborhood of $K$. That is to say, there exist a neighborhood $V$ of $K$ and complex numbers $a_{n}=a_{n}(f)$ such that for every $z$ in $V$ we have

$$
f(z)=\sum_{n=0}^{\infty} a_{n} F_{n}(z) .
$$

Example 2.2. Let $R>1$ and $K$ be the compact delimited by the ellipse with the foci at the points 1 and -1 and semi-axes

$$
a=\frac{1}{2}(R+1 / R) \quad \text { and } \quad b=\frac{1}{2}(R-1 / R) .
$$

The equation of this ellipse can be written in the form

$$
z=\frac{1}{2}\left(R e^{i \theta}+\frac{1}{R e^{i \theta}}\right), \quad 0 \leq \theta<2 \pi .
$$

Hence, the exterior conformal map associated with $K$ is given by

$$
\psi(w)=\frac{1}{2}\left(R w+\frac{1}{R w}\right) .
$$

The corresponding Faber polynomials are

$$
F_{n}(z)=\frac{2}{R^{n}} C_{n}(z), \quad(n \geq 1)
$$

where $C_{n}$ is the Chebychev polynomial of order $n$,

$$
C_{n}(t)=\cos (n \arccos (t)), \quad(t \in[-1 ; 1]) .
$$


We also notice that, for any $n \geq 1$, we have

$$
F_{n}(\psi(w))=w^{n}+\frac{1}{R^{2 n} w^{n}} .
$$

2.2. Consequences of the regularity of the boundary $\Gamma$. If $\Gamma$, the boundary of $K$, is a Jordan curve, its regularity influences the behaviour of the map $\psi$ and the asymptotic behaviour of the sequence of Faber polynomials $\left(F_{n}\right)_{n \geq 0}$ on $\Gamma$.

A function $\rho: \mathbb{R}^{+} \longrightarrow \mathbb{R}^{+}$is called a modulus of continuity if it satisfies:

(1) $\rho$ is increasing;

(2) $\lim _{t \rightarrow 0} \rho(t)=0$;

(3) $\rho$ is subadditive, i.e.,

$$
\rho(t+s) \leq \rho(t)+\rho(s) .
$$

A modulus of continuity is said to satisfy the Alper condition if

$$
\int_{0}^{\varepsilon} \frac{\rho(x)}{x} \log \left(\frac{1}{x}\right) d x<\infty
$$

for some $\varepsilon>0$.

For a fixed modulus of continuity $\rho$, a Jordan curve $\Gamma$ is said to be of class $C^{\rho}$ if it has a parametrization $\tau:[0 ; 1) \longrightarrow \Gamma$ that is differentiable, with $\tau^{\prime}(x) \neq 0$ of all $x \in[0,1)$ and $\tau^{\prime}$ satisfying the continuity condition

$$
\left|\tau^{\prime}\left(x_{1}\right)-\tau^{\prime}\left(x_{2}\right)\right| \leq \rho\left(\left|x_{1}-x_{2}\right|\right), \quad x_{1}, x_{2} \in[0 ; 1) .
$$

A Jordan arc is of class $C^{\rho}$ if it is a subarc of a Jordan curve of class $C^{\rho}$.

The following theorem is a simplified version of [15, Theorem 1.1].

Theorem 2.3 ([15]). Let $\Gamma$ be a rectifiable Jordan curve. For $z_{0} \in \Gamma$, assume that $\Gamma$ has an exterior angle of opening $\alpha \pi$ at $z_{0}$, with $0<\alpha \leq 2$, formed by $C^{\rho}$ arcs, where $\rho$ is a modulus of continuity satisfying the Alper condition. Then,

$$
F_{n}\left(z_{0}\right)=\alpha \phi\left(z_{0}\right)^{n}+o(1), \quad \text { as } n \rightarrow \infty .
$$

Remark 2.4. In the above result, the case $\alpha=1$ corresponds to a point where the boundary form an arc of regularity $C^{\rho}$, the (excluded) case $\alpha=0$ to an inside pointing cusp and the case $\alpha=2$ to an outside pointing cusp.

It is often sufficient to consider $\rho(x)=x^{\beta}$ for some $\beta>0$. The class $C^{\rho}$ is then the Hölder class $C^{1, \beta}$.

The following definition will be used in the next sections as a basic geometric condition about the regularity of the boundary.

Definition 2.5. A rectifiable Jordan curve $C$ will be called an Alper curve with (possible) angles if for all $\zeta \in C$ either $C$ forms an arc of regularity $C^{\rho}$ in a neighborhood of $\zeta$, or $C$ has an exterior angle of opening $\alpha \pi$ at $\zeta, 0<\alpha \leq 2$, formed by $C^{\rho}$ arcs, where $\rho$ is a modulus of continuity satisfying the Alper condition. 
We want to stress here that Alper curves with angles do not have inside pointing cusps.

Remark 2.6. According to Theorem 2.3, if $\Gamma$ is an Alper curve with (possible) angles, then for any $\zeta \in \Gamma$, the sequence $\left(\left|F_{n}(\zeta)\right|\right)_{n \geq 0}$ is eventually bounded below by a positive number.

We also record for further use the following classical result about analytic Jordan curves and an inequality of Kövari and Pommerenke [10].

Theorem 2.7. Suppose that $\Gamma$ is an analytic Jordan curve. Then $\psi$ has an analytic univalent extension in $\overline{r \mathbb{D}}^{c}$ for some $r \in(0 ; 1)$.

Theorem 2.8. [10] If $K$ is convex, then for all $z \in \Gamma$ and for all $n \geq 0$,

$$
\left|F_{n}(z)-\phi(z)^{n}\right| \leq 1
$$

\subsection{Faber polynomials and operators on a Banach space.}

Notation. For a complex Banach space $\mathcal{X}$, we denote by $B(\mathcal{X})$ the Banach algebra of the bounded linear operators from $\mathcal{X}$ into itself and by $I=I_{\mathcal{X}}$ the identity on $\mathcal{X}$. The spectrum $\sigma(T)$ of $T \in B(\mathcal{X})$ is defined ([5]) by

$$
\sigma(T):=\{\lambda \in \mathbb{C} ;(T-\lambda I) \text { is not invertible in } B(\mathcal{X})\} .
$$

Lemma 2.9. Let $\mathcal{X}$ be a Banach space. Let $T \in B(\mathcal{X})$ be such that $\sigma(T) \subset K$. Then, for $\lambda \in \mathbb{C},|\lambda|>1$, we have

$$
(\psi(\lambda) I-T)^{-1}=\psi^{\prime}(\lambda)^{-1} \sum_{n=0}^{\infty} \lambda^{-n-1} F_{n}(T) .
$$

Proof. Let $\lambda \in \mathbb{C},|\lambda|>1$. Let $R=|\lambda|$. Define

$$
f(z)=\frac{\psi^{\prime}(\lambda)}{\psi(\lambda)-z}, \quad z \in G_{R}
$$

It follows from (2.1) that for every $z \in G_{R}$ we have

$$
f(z)=\sum_{n=0}^{\infty} \lambda^{-n-1} F_{n}(z) .
$$

As $f$ is an analytic function on $G_{R}$, we have from Theorem 2.1 the uniform convergence of the series in any compact subset of $G_{R}$.

Using $\sigma(T) \subset K$, the holomorphic functional calculus gives

$$
(\psi(\lambda) I-T)^{-1}=\psi^{\prime}(\lambda)^{-1} \sum_{n=0}^{\infty} \lambda^{-n-1} F_{n}(T) .
$$


In their article [1], A. Atzmon, A. Eremenko and M. Sodin have proved that for any compact set $K \subset \mathbb{C}$ with connected complement, there exists a sequence of polynomials $\left(P_{n}\right)_{n \geq 0}$ and a positive number $r$ such that if $T$ is a bounded operator on $\mathcal{X}$, its spectrum is included in $K$ if and only if

$$
\limsup _{n \rightarrow \infty}\left\|P_{n}(T)\right\|^{1 / n} \leq r .
$$

If $K$ is connected, this can be expressed in terms of Faber polynomials.

Theorem 2.10 ([1]). Let $\mathcal{X}$ be a Banach space and $T \in B(\mathcal{X})$. Then $\sigma(T) \subset K$ if and only if

$$
\limsup _{n \rightarrow \infty}\left\|F_{n}(T)\right\|^{1 / n} \leq 1
$$

\section{Condition on the Weak Faber orbits}

Let $K$ be a simply connected compact subset of the complex plane with a non empty interior. The aim of this section is to propose some conditions implying that $\sigma(T)$ is included in the interior of $K$. Such results have been proved in the case when $K$ is the closed unit disc by Mlak [11], Weiss [19], van Neerven [18] and Nikolski $[13,14]$.

Let $\left(F_{n}\right)_{n \geq 0}$ be the sequence of Faber polynomials associated with $K$.

Notation. The set of finitely supported sequences will be denoted $\mathbb{C}^{(\mathbb{N})}$ and $\left(e_{n}\right)_{n \geq 0}$ will denote the canonical algebraic basis of $\mathbb{C}^{(\mathbb{N})}$. The characteristic function of a set $A$ will be denoted by $\chi_{A}$.

Definition 3.1. A Banach space $\mathcal{E} \subset \mathbb{C}^{\mathbb{N}}$ is said to be an admissible sequence space if it satisfies the following properties:

- if $\left|a_{n}\right| \leq\left|b_{n}\right|$ for all $n \in \mathbb{N}$ and $\left(b_{n}\right)_{n \geq 0} \in \mathcal{E}$, then $\left(a_{n}\right)_{n \geq 0} \in \mathcal{E}$ and $\left\|\left(a_{n}\right)_{n \geq 0}\right\|_{\mathcal{E}} \leq\left\|\left(b_{n}\right)_{n \geq 0}\right\|_{\mathcal{E}}$

- for all $k \in \mathbb{N}$ there exists a sequence $\left(a_{n}\right)_{n \geq 0} \in \mathcal{E}$ such that $a_{k} \neq 0$.

Proposition 3.2. Let $\mathcal{E}$ be an admissible sequence space. Then $\mathbb{C}^{(\mathbb{N})} \subset \mathcal{E}$.

Proof. Let $k \in \mathbb{N}$. There exists a sequence $\left(a_{n}\right)_{n>0} \in \mathcal{E}$ such that $a_{k} \neq 0$ by the second condition of admissibility. Then $\left|\chi_{\{k\}}(n)\right| \leq\left|a_{k}\right|^{-1}\left|a_{n}\right|$ for all $n \in \mathbb{N}$. It follows then from the first condition of admissibility that $\chi_{\{k\}}$ belongs to $\mathcal{E}$. By taking finite linear combinations, we get that the finitely supported sequences belong to $\mathcal{E}$.

The following definition extends the corresponding unit-disc analogue from [14].

Definition 3.3. Let $\mathcal{E} \subset \mathbb{C}^{\mathbb{N}}$ be a Banach space and $T \in B(\mathcal{X})$. The operator $T$ is said to be of weak type $\mathcal{E}$ if for every pair $\left(x ; x^{*}\right) \in \mathcal{X} \times \mathcal{X}^{*}$ we have

$$
\left(\left\langle F_{n}(T) x ; x^{*}\right\rangle\right)_{n \geq 0} \in \mathcal{E} \text {. }
$$

Theorem 3.5 below, which is the main result of this section, is a generalisation of the following result of J. van Neerven [18]. 
Theorem 3.4 ([18]). Let $\mathcal{X}$ be a Banach space, $T \in B(\mathcal{X})$ and $\mathcal{E}$ an admissible sequence space such that for any $\zeta \in \mathbb{T}$

$$
\left\|\left(1, \zeta, \zeta^{2} \ldots, \zeta^{N}, 0,0, \ldots\right)\right\|_{\mathcal{E}} \underset{N \rightarrow \infty}{\longrightarrow} \infty .
$$

If $T$ is of weak type $\mathcal{E}$, then

$$
r(T)<1 .
$$

Theorem 3.5. Let $\mathcal{X}$ be a Banach space, $T \in B(\mathcal{X})$ and $\mathcal{E}$ an admissible sequence space such that for any $\zeta \in \partial K$

$$
\left\|\left(F_{0}(\zeta), \ldots, F_{N}(\zeta), 0,0, \ldots\right)\right\|_{\mathcal{E}} \underset{N \rightarrow \infty}{\longrightarrow} \infty .
$$

If $T$ is of weak type $\mathcal{E}$, then

$$
\sigma(T) \subset \operatorname{int}(K)
$$

Lemma 3.6. Let $\mathcal{E} \subset \mathbb{C}^{\mathbb{N}}$ be a Banach space such that for any $k \in \mathbb{N}$, the map $\left(a_{n}\right)_{n \geq 0} \mapsto a_{k}$ is bounded. Suppose that $T \in B(\mathcal{X})$ is a weak type $\mathcal{E}$ operator. Then

$$
\sup \left\{\left\|\left(\left\langle F_{n}(T) x ; x^{*}\right\rangle\right)_{n \geq 0}\right\|_{\mathcal{E}} ;\left\|x^{*}\right\| \leq 1,\|x\| \leq 1\right\}<\infty .
$$

Proof. Let us prove that for any $x \in \mathcal{X}$, the map

$$
\begin{aligned}
f_{x}: \mathcal{X}^{*} & \rightarrow \mathcal{E} \\
x^{*} & \mapsto\left(\left\langle F_{n}(T) x ; x^{*}\right\rangle\right)_{n \geq 0}
\end{aligned}
$$

is bounded.

Indeed, $f_{x}$ is well defined because $T$ is a weak type $\mathcal{E}$ operator. Let $\left(x_{n}^{*}\right)_{n \geq 0} \subset$ $\mathcal{X}^{*}, x^{*} \in \mathcal{X}^{*}$ and $r \in \mathcal{E}$ be such that

$$
x_{n}^{*} \rightarrow x^{*} \text { and } f_{x}\left(x_{n}^{*}\right) \stackrel{\mathcal{E}}{\longrightarrow} r .
$$

We infer from the boundedness of the maps $\left(a_{n}\right)_{n \geq 0} \mapsto a_{k}$, that for any $k \in \mathbb{N}$

$$
\left[f_{x}\left(x_{n}^{*}\right)\right]_{k} \underset{n \rightarrow \infty}{\longrightarrow} r_{k}
$$

where $\left[f_{x}\left(x_{n}^{*}\right)\right]_{k}$ denotes the $k$-th element of the sequence $f_{x}\left(x_{n}^{*}\right)$. For any $k \in \mathbb{N}$

$$
\left[f_{x}\left(x_{n}^{*}\right)\right]_{k}=\left\langle F_{k}(T) x ; x_{n}^{*}\right\rangle \underset{n \rightarrow \infty}{\longrightarrow}\left\langle F_{k}(T) x ; x^{*}\right\rangle=\left[f_{x}\left(x^{*}\right)\right]_{k} .
$$

Thus $f_{x}\left(x^{*}\right)=r$ and, according to the closed graph theorem [5], $f_{x}$ is bounded. In particular, for any $x \in \mathcal{X}$

$$
\sup \left\{\left\|\left(\left\langle F_{n}(T) x ; x^{*}\right\rangle\right)_{n \geq 0}\right\|_{\mathcal{E}} ;\left\|x^{*}\right\| \leq 1\right\}<\infty
$$

For any $x^{*} \in \mathcal{X}^{*}$ let

$$
\begin{aligned}
g_{x^{*}}: \mathcal{X} & \rightarrow \mathcal{E} \\
x & \mapsto f_{x}\left(x^{*}\right) .
\end{aligned}
$$

Then, in the same way as for $f_{x}$, we can prove that, for every $x^{*}$, the map $g_{x^{*}}$ is bounded. Using (3.1) we obtain that

$$
\sup _{\left\|x^{*}\right\| \leq 1}\left\|g_{x^{*}}(x)\right\|<\infty
$$


for an arbitrary $x \in \mathcal{X}$. Using the uniform boundedness principle [5] we infer that $\left\{g_{x^{*}}\right\}_{\left\|x^{*}\right\| \leq 1}$ is a bounded set. Thus

$$
\sup \left\{\left\|\left(\left\langle F_{n}(T) x ; x^{*}\right\rangle\right)_{n \geq 0}\right\|_{\mathcal{E}} ;\left\|x^{*}\right\| \leq 1,\|x\| \leq 1\right\}<\infty,
$$

which completes the proof.

Lemma 3.7. Let $T \in B(\mathcal{X})$ be a linear operator acting on the Banach space $\mathcal{X}$. For all $\lambda \in \partial \sigma(T)$, all $\varepsilon>0$ and all $N \in \mathbb{N}$ there exist $x_{N} \in \mathcal{X}$ and $x_{N}^{*} \in \mathcal{X}^{*}$ satisfying $\left\|x_{N}\right\|=1,\left\|x_{N}^{*}\right\|=1$ and

$$
\left|\left\langle F_{n}(T) x_{N} ; x_{N}^{*}\right\rangle\right|>\left|F_{n}(\lambda)\right|-\varepsilon, \quad n=0,1, \ldots N .
$$

Proof. Let $\lambda \in \partial \sigma(T), \varepsilon>0$ and $N \in \mathbb{N}$. As $\lambda \in \partial \sigma(T)$, we have that $\lambda$ belongs to $\sigma_{\text {app }}(T)$, the approximate point spectrum of $T$ (cf. [5, Chapter 7.6]). By definition, this means that there exists a sequence $\left(y_{n}\right)_{n \geq 0}$ in $\mathcal{X}$ such that $\left\|y_{n}\right\|=1$ for all $n \in \mathbb{N}$ and

$$
\left\|T y_{n}-\lambda y_{n}\right\| \underset{n \rightarrow \infty}{\longrightarrow} 0
$$

Thus, for each fixed $k \in \mathbb{N}$,

$$
\lim _{n \rightarrow \infty}\left\|F_{k}(T) y_{n}-F_{k}(\lambda) y_{n}\right\|=0 .
$$

Let $n_{1}$ be such that

$$
\left\|F_{k}(T) y_{n_{1}}-F_{k}(\lambda) y_{n_{1}}\right\|<\varepsilon, \quad \forall k=0,1, \ldots N .
$$

Let $x_{N}=y_{n_{1}}$ and $x_{N}^{*} \in \mathcal{X}^{*}$ be such that $\left\|x_{N}^{*}\right\|=1$ and $\left\langle x_{N}^{*} ; x_{N}\right\rangle=1$. Then for $k \in\{0,1, \ldots N\}$, we have

$$
\begin{aligned}
\left|\left\langle F_{k}(T) x_{N} ; x_{N}^{*}\right\rangle\right| & \geq\left|\left\langle F_{k}(\lambda) x_{N} ; x_{N}^{*}\right\rangle\right|-\left|\left\langle F_{k}(T) x_{N}-F_{k}(\lambda) x_{N} ; x_{N}^{*}\right\rangle\right| \\
& >\left|F_{k}(\lambda)\right|-\varepsilon .
\end{aligned}
$$

Proof of Theorem 3.5. From Lemma 3.6 we know that there exists $M \geq 0$ such that for any $x \in \mathcal{X}, x^{*} \in \mathcal{X}^{*}$, with $\|x\|=1$ and $\left\|x^{*}\right\|=1$, we have

$$
\left\|\left(\left\langle F_{n}(T) x ; x^{*}\right\rangle\right)_{n \geq 0}\right\|_{\mathcal{E}} \leq M .
$$

Suppose that $\sigma(T) \nsubseteq \operatorname{int}(K)$, then there exists $\lambda \in \partial \sigma(T) \backslash \operatorname{int}(K)$. From Lemma 3.7 we get that for every $\varepsilon>0$ and $N \in \mathbb{N}$ there exist $x_{N} \in \mathcal{X}$ and $x_{N}^{*} \in \mathcal{X}^{*}$ satisfying $\left\|x_{N}\right\| \leq 1,\left\|x_{N}^{*}\right\| \leq 1$ and

$$
\left|\left\langle F_{n}(T) x_{N} ; x_{N}^{*}\right\rangle\right|>\left|F_{n}(\lambda)\right|-\varepsilon, \quad n=0,1, \ldots N .
$$

Therefore, $\left|\left\langle F_{n}(T) x_{N} ; x_{N}^{*}\right\rangle\right| \geq\left|F_{n}(\lambda)\right| \chi_{\{0, \ldots N\}}(n)-\varepsilon \chi_{\{0, \ldots N\}}(n)$ for all $n$. Thus

$$
\left\|\left(\left\langle F_{n}(T) x_{N} ; x_{N}^{*}\right\rangle\right)_{n \geq 0}\right\|_{\mathcal{E}} \geq\left\|\left(F_{0}(\zeta), \ldots, F_{N}(\zeta), 0,0, \ldots\right)\right\|_{\mathcal{E}}-\varepsilon\left\|\chi_{\{0, \ldots N\}}\right\|_{\mathcal{E}} .
$$

We obtain that for any $\varepsilon>0$ and $N \in \mathbb{N}$

$$
\left\|\left(F_{0}(\zeta), \ldots, F_{N}(\zeta), 0,0, \ldots\right)\right\|_{\mathcal{E}}-\varepsilon\left\|\chi_{\{0, \ldots N\}}\right\|_{\mathcal{E}} \leq M
$$


Hence, for every $N \in \mathbb{N}$ we have

$$
\left\|\left(F_{0}(\zeta), \ldots, F_{N}(\zeta), 0,0, \ldots\right)\right\|_{\mathcal{E}} \leq M,
$$

which gives the desired contradiction if $\lambda \in \partial K$. If $\lambda \notin K$, then

$$
\left|F_{n}(\lambda)\right|^{1 / n} \underset{n \rightarrow \infty}{\longrightarrow} R>1 .
$$

But we know that for any $\zeta \in \partial K, \lim \sup _{n \rightarrow \infty}\left|F_{n}(\zeta)\right|^{1 / n} \leq 1$, and

$$
\left\|\left(F_{0}(\zeta), \ldots, F_{N}(\zeta), 0,0, \ldots\right)\right\|_{\mathcal{E}} \underset{N \rightarrow \infty}{\longrightarrow} \infty .
$$

Thus, if $\lambda \notin K$ we get from the first condition of admissibility that

$$
\left\|\left(F_{0}(\zeta), \ldots, F_{N}(\zeta), 0,0, \ldots\right)\right\|_{\mathcal{E}} \underset{N \rightarrow \infty}{\longrightarrow} \infty
$$

which gives a contradiction.

We now give some illustrating examples; we start with one based on weighted $l^{p}$ spaces.

Corollary 3.8. Let $1 \leq p<\infty$ and let $\left(\omega_{n}\right)_{n \geq 0}$ be a sequence of non negative numbers. We suppose that for any $\zeta \in \partial K$,

$$
\sum_{n=0}^{\infty} \omega_{n}\left|F_{n}(\zeta)\right|^{p}=\infty .
$$

Let $\mathcal{X}$ be a Banach space. Let $T \in B(\mathcal{X})$ be such that for every $\left(x, x^{*}\right) \in \mathcal{X} \times \mathcal{X}^{*}$ we have

$$
\sum_{n=0}^{\infty} \omega_{n}\left|\left\langle F_{n}(T) x ; x^{*}\right\rangle\right|^{p}<\infty .
$$

Then $\sigma(T) \subset \operatorname{int}(K)$.

Corollary 3.9. Let $\left(\omega_{n}\right)_{n \geq 0}$ be a sequence of non negative numbers. We suppose that for any $\zeta \in \partial K$,

$$
\limsup _{n \rightarrow \infty} \omega_{n}\left|F_{n}(\zeta)\right|=\infty
$$

Let $\mathcal{X}$ be a Banach space. Let $T \in B(\mathcal{X})$ be such that for every $\left(x, x^{*}\right) \in \mathcal{X} \times \mathcal{X}^{*}$ we have

$$
\sup _{n \geq 0} \omega_{n}\left|\left\langle F_{n}(T) x ; x^{*}\right\rangle\right|<\infty
$$

Then $\sigma(T) \subset \operatorname{int}(K)$.

Remark 3.10. According to Remark 2.6, if $\partial K$ is an Alper curve with (possible) angles, the conditions on the sequence $\left(\omega_{n}\right)_{n \geq 0}$ can be replaced by

$$
\sum_{n=0}^{\infty} \omega_{n}=\infty
$$

in Corollary 3.8 and by

$$
\limsup _{n \rightarrow \infty} \omega_{n}=\infty
$$

in Corollary 3.9. 
Proof of Corollaries 3.8 and 3.9. We have that

$$
\lim _{|z| \rightarrow \infty}|\phi(z)|=\infty .
$$

Therefore, there exists a $R>1$ such that

$$
\sigma(T) \cap\{z:|\phi(z)| \geq R\}=\varnothing .
$$

Thus, we get from Theorem 2.10 that

$$
\limsup _{n \rightarrow \infty}\left\|F_{n}(T)\right\|^{1 / n}<R .
$$

Let $\left(\tilde{\omega}_{n}\right)_{n \geq 0}$ be the positive sequence defined by

$$
\tilde{\omega}_{n}= \begin{cases}\omega_{n} & \text { if } \omega_{n} \neq 0 \\ R^{-n p} & \text { if } \omega_{n}=0\end{cases}
$$

For every $\left(x, x^{*}\right) \in \mathcal{X} \times \mathcal{X}^{*}$ we have

$$
\sum_{n=0}^{\infty} \tilde{\omega}_{n}\left|\left\langle F_{n}(T) x ; x^{*}\right\rangle\right|^{p}<\infty .
$$

If $p<\infty$, then the space

$$
l^{p}(\tilde{\omega}):=\left\{\left(a_{n}\right)_{n \geq 0} ; \sum_{n=0}^{\infty} \tilde{\omega}_{n}\left|a_{n}\right|^{p}<\infty\right\}
$$

with the norm:

$$
\left\|\left(a_{n}\right)_{n \geq 0}\right\|_{l^{p}(\tilde{\omega})}=\left(\sum_{n=0}^{\infty} \tilde{\omega}_{n}\left|a_{n}\right|^{p}\right)^{1 / p}
$$

is an admissible sequence space and the condition

$$
\left\|\left(F_{0}(\zeta), \ldots, F_{N}(\zeta), 0,0, \ldots\right)\right\|_{l^{p}(\tilde{\omega})} \underset{N \rightarrow \infty}{\longrightarrow} \infty
$$

is equivalent to

$$
\sum_{n=0}^{\infty} \tilde{\omega}_{n}\left|F_{n}(\zeta)\right|^{p}=\infty
$$

If $p=\infty$, then the space

$$
l^{\infty}(\tilde{\omega})=\left\{\left(a_{n}\right)_{n \geq 0} ; \sup _{n \geq 0} \tilde{\omega}_{n}\left|a_{n}\right|<\infty\right\}
$$

with the norm

$$
\left\|\left(a_{n}\right)_{n \geq 0}\right\|_{l^{\infty}(\tilde{\omega})}=\sup _{n \geq 0} \tilde{\omega}_{n}\left|a_{n}\right|
$$

is an admissible sequence space and the condition

$$
\left\|\left(F_{0}(\zeta), \ldots, F_{N}(\zeta), 0,0, \ldots\right)\right\|_{l^{p}(\tilde{\omega})} \underset{N \rightarrow \infty}{\longrightarrow} \infty
$$

is equivalent to

$$
\limsup _{n \rightarrow \infty} \tilde{\omega}_{n}\left|F_{n}(\zeta)\right|=\infty
$$


It is then sufficient to apply Theorem 3.5 with the space $\mathcal{E}=l^{p}(\tilde{\omega})$ to conclude.

We give now another application of Theorem 3.5 based on Orlicz spaces.

Definition 3.11. A function $\Phi:[0 ; \infty) \longrightarrow[0 ; \infty)$ is a Young function if it is convex and it satisfies

$$
\frac{\Phi(x)}{x} \underset{x \rightarrow 0}{\longrightarrow} 0 \text { and } \frac{\Phi(x)}{x} \underset{x \rightarrow \infty}{\longrightarrow} \infty .
$$

For any complex sequence $\left(x_{n}\right)_{n \geq 0}$ we set

$$
M_{\Phi}(x)=\sum_{n=0}^{\infty} \Phi\left(\left|x_{n}\right|\right) .
$$

The Orlicz space $L^{\Phi}$ is the space of all sequences $\left(x_{n}\right)_{n \geq 0}$ such that there exists $k>0$ such that $M_{\Phi}(k x)<\infty$.

Theorem 3.12. Let $\Phi$ be a Young function, the Orlicz space $L^{\phi}$ with the norm

$$
\left\|\left(x_{n}\right)_{n \geq 0}\right\|_{L^{\Phi}}=\inf \left\{k ; M_{\Phi}\left(\frac{1}{k} x\right) \leq 1\right\}
$$

is a Banach space and if $\Phi(t)>0$ for all $t>0$, then

$$
\left\|\chi_{\{0, \ldots, n-1\}}\right\|_{L^{\Phi}} \underset{n \rightarrow \infty}{\longrightarrow} \infty \text {. }
$$

For the proof we use the following useful result.

Lemma 3.13 ([18]). Let $\varphi: \mathbb{R}_{+} \longrightarrow \mathbb{R}_{+}$be non-decreasing with $\varphi(t)>0$ for all $t>0$. Then there exists a Young function $\Phi$ such that the Orlicz space $L^{\Phi}$ contains every sequence $\left(a_{n}\right)_{n \geq 0}$ such that

$$
\sum_{n \geq 0} \varphi\left(\left|a_{n}\right|\right)<\infty
$$

and satisfies

$$
\left\|\chi_{\{0, \ldots, n-1\}}\right\|_{L^{\Phi}} \underset{n \rightarrow \infty}{\longrightarrow} \infty .
$$

Proof. Replacing $\varphi$ by a multiple of $\varphi$ we may assume that $\varphi(1)=1$. Let $\Phi$ be defined by

$$
\Phi(t)=\int_{0}^{t} \varphi(s) d s .
$$

Then $\Phi$ is a Young function and if $\left(a_{n}\right)_{n \geq 0}$ is such that

$$
\sum_{n \geq 0} \varphi\left(\left|a_{n}\right|\right)<\infty
$$

then the set of indexes $n$ such that $\left|a_{n}\right|>1$ is finite and if $\left|a_{n}\right| \leq 1$, then $\Phi\left(\left|a_{n}\right|\right) \leq \varphi\left(\left|a_{n}\right|\right)$. Thus we get

$$
\sum_{n \geq 0} \Phi\left(\left|a_{n}\right|\right)<\infty
$$


so $\left(a_{n}\right)_{n \geq 0} \in L^{\Phi}$.

But $\Phi$ is strictly positive and increasing on $(0 ; \infty)$, therefore we obtain

$$
\left\|\chi_{\{0, \ldots, n-1\}}\right\|_{L^{\Phi}}=\inf \left\{k ; \sum_{k=0}^{n-1} \Phi(1 / k) \leq 1\right\} \underset{n \rightarrow \infty}{\longrightarrow} \infty .
$$

As Orlicz spaces of sequences are admissible sequence spaces we obtain the following result.

Corollary 3.14. We suppose that $\left(\left|F_{n}(\zeta)\right|\right)_{n \geq 0}$ is eventually bounded below by a positive number for every $\zeta \in \partial K$. Let $T \in B(\mathcal{X})$ and let $\varphi: \mathbb{R}_{+} \longrightarrow \mathbb{R}_{+}$be a non-decreasing function with $\varphi(t)>0$ for all $t>0$. If for every $x \in \mathcal{X}$ and $x^{*} \in \mathcal{X}^{*}$

$$
\sum_{n=0}^{\infty} \varphi\left(\left|\left\langle F_{n}(T) x ; x^{*}\right\rangle\right|\right)<\infty
$$

then

$$
\sigma(T) \subset \operatorname{int}(K)
$$

Remark 3.15. According to Remark 2.6, the above condition about the sequence $\left(\left|F_{n}(\zeta)\right|\right)_{n>0}$ is automaticaly satisfied whenever $\partial K$ is an Alper curve with (possible) angles.

Definition 3.16. The function $\varphi$ is said to satisfy the $\Delta_{2}$-condition at 0 if there exist $\varepsilon>0$ and $K>0$ such that for any $x \in[0 ; \varepsilon]$, we have $\varphi(t / 2) \geq K \varphi(t)$.

Corollary 3.17. We suppose that $\left(\left|F_{n}(\zeta)\right|\right)_{n \geq 0}$ is eventually bounded below by a positive number for every $\zeta \in \partial K$. Let $T \in B(\mathcal{X})$, let $\varphi: \mathbb{R}_{+} \longrightarrow \mathbb{R}_{+}$be a non-decreasing function satisfying the $\Delta_{2}$-condition at 0 with $\varphi(t)>0$ for $t>0$, and let $\left(\omega_{n}\right)_{n>0}$ be a sequence of non negative numbers such that

$$
\sum_{n=0}^{\infty} \omega_{n} \varphi\left(\omega_{n}\right)=\infty
$$

If for every $x \in \mathcal{X}$ and $x^{*} \in \mathcal{X}^{*}$

$$
\sum_{n=0}^{\infty} \varphi\left(\omega_{n}\left|\left\langle F_{n}(T) x ; x^{*}\right\rangle\right|\right)<\infty
$$

then

$$
\sigma(T) \subset \operatorname{int}(K)
$$

Proof. Adapting the proof of Corollary 3.8, we may suppose $\omega_{n}$ to be positive for every $n$. Replacing if necessary $\omega_{n}$ by $\min \left(\omega_{n}, \varepsilon\right)$, we may also assume that $\omega_{n} \in(0 ; \varepsilon]$ for each $n$. Let $\Phi$ be as in Lemma 3.13 and let $\mathcal{E}$ be the space

$$
\mathcal{E}=\left\{\left(x_{n}\right)_{n \geq 0} ;\left(\omega_{n} x_{n}\right)_{n \geq 0} \in L^{\Phi}\right\}
$$

with the norm

$$
\left\|\left(x_{n}\right)_{n \geq 0}\right\|_{\mathcal{E}}=\left\|\left(\omega_{n} x_{n}\right)_{n \geq 0}\right\|_{L^{\Phi}} .
$$


The $\Delta_{2}$ condition gives that for any $t \in(0 ; \varepsilon]$,

$$
\Phi(t)=\int_{0}^{t} \varphi(s) d s \geq \frac{t}{2} \varphi(t / 2) \geq \frac{K t}{2} \varphi(t) .
$$

Let $k>1$, and $m$ be the integer part of $(\ln (k) / \ln (2)+1)$. Then

$$
\sum_{j=0}^{n-1} \Phi\left(\omega_{j} / k\right) \geq \frac{K^{m}}{2^{m}} \sum_{j=0}^{n-1} \omega_{j} \varphi\left(\omega_{j}\right)
$$

This proves that

$$
\left\|\chi_{\{0, \ldots, n-1\}}\right\|_{\mathcal{E}} \underset{n \rightarrow \infty}{\longrightarrow} \infty
$$

An application of Theorem 3.5 completes the proof.

Corollary 3.18. We suppose that $\left(\left|F_{n}(\zeta)\right|\right)_{n \geq 0}$ is eventually bounded below by a positive number for every $\zeta \in \partial K$. Let $T \in B(\mathcal{X})$. Suppose there exists a positive function $\varphi$ on $[0 ; 1)$ such that

$$
\sqrt{1-r} \varphi(r) \underset{r \rightarrow 1}{\longrightarrow} 0
$$

and such that

$$
\frac{1}{\varphi(r)}\left\|\left(r^{n}\left\langle F_{n}(T) x ; x^{*}\right\rangle\right)_{n \geq 0}\right\|_{2} \underset{r \rightarrow 1}{\longrightarrow} 0,
$$

for every $x \in \mathcal{X}$ and every $x^{*} \in \mathcal{X}^{*}$. Then

$$
\sigma(T) \subset \operatorname{int}(K) \text {. }
$$

Lemma 3.19. Let $\varphi$ be a positive function on $[0 ; 1)$ such that $\varphi(r)$ tends to infinity and $\sqrt{1-r} \varphi(r)$ tends to 0 as $r$ tends to 1 . Then the space $\mathcal{E}$ defined by

$$
\mathcal{E}=\left\{\left(x_{n}\right)_{n \geq 0} ; \frac{1}{\varphi(r)}\left\|\left(r^{n} x_{n}\right)_{n \geq 0}\right\|_{2} \underset{r \rightarrow 1}{\longrightarrow} 0\right\},
$$

with the norm

$$
\left\|\left(x_{n}\right)_{n \geq 0}\right\|_{\mathcal{E}}=\sup _{r \in(0 ; 1)} \frac{1}{\varphi(r)}\left\|\left(r^{n} x_{n}\right)_{n \geq 0}\right\|_{2},
$$

is an admissible Banach function space over $\mathbb{N}$ such that

$$
\left\|\chi_{\{0, \ldots, n\}}\right\|_{\mathcal{E}} \underset{n \rightarrow \infty}{\longrightarrow} \infty \text {. }
$$

Proof. It is easy to see that $\mathcal{E}$ is an admissible sequence space. Furthermore, we have

$$
\left\|\chi_{\{0, \ldots, n-1\}}\right\|_{\mathcal{E}}=\sup _{r \in(0 ; 1)} \frac{1}{\varphi(r)}\left\|\left(1, r, r^{2}, \ldots, r^{n-1}, 0,0, \ldots\right)\right\|_{2},
$$

and

$$
\left\|\left(1, r, r^{2}, \ldots, r^{n-1}, 0,0, \ldots\right)\right\|_{2}^{2}=\sum_{k=0}^{n-1} r^{2 k}=\frac{1-r^{2 n}}{1-r^{2}}
$$


Therefore,

$$
\begin{aligned}
\frac{1}{\varphi(r)}\left\|\left(1, r, r^{2}, \ldots, r^{n-1}, 0,0, \ldots\right)\right\|_{2} & =\frac{1}{\varphi(r)} \frac{\sqrt{1-r^{n}}}{\sqrt{1-r}} \frac{\sqrt{1+r^{n}}}{\sqrt{1+r}} \\
& \geq \frac{1}{2} \frac{\sqrt{1-r^{n}}}{\varphi(r) \sqrt{1-r}} .
\end{aligned}
$$

Let $M>0$. As $\sqrt{1-r} \varphi(r)$ tends to 0 as $r$ tends to 1 , there exists $r_{0}<1$ such that

$$
\frac{1}{\varphi\left(r_{0}\right) \sqrt{1-r_{0}}}>4 M
$$

Let $N$ be such that $\sqrt{1-r_{0}^{N}}>1 / 2$. We get that for each $n \geq N$ we have

$$
\left\|\chi_{\{0, \ldots, n-1\}}\right\|_{\mathcal{E}} \geq M
$$

The proof is complete.

Proof of Corollary 3.18. Replacing, if necessary, $\varphi(r)$ by $\max \left(\varphi(r) ;(1-r)^{-1 / 4}\right)$, we may assume that $\varphi(r) \underset{r \rightarrow 1}{\longrightarrow} \infty$. We can then apply Lemma 3.19 and Theorem 3.5 to obtain the inclusion $\sigma(T) \subset \operatorname{int}(K)$.

\section{Condition on the Weak Resolvent}

The purpose of this section is, as in the previous one, to find conditions assuring spectral inclusion in the interior of a given simply connected compact set $K$. We suppose here that the spectrum of the bounded linear operator under consideration is included in the compact set $K$. Nikolski [13] called conditions of this type Tauberian conditions for the spectral radius. The main interest of this approach is that it is well adapted to conditions expressed in terms of the weak resolvent, that is to say on the functions

$$
z \notin K \mapsto\left\langle(z I-T)^{-1} x ; x^{*}\right\rangle,
$$

where $x \in \mathcal{X}$ and $x^{*} \in \mathcal{X}^{*}$.

We recall that $\left(F_{n}\right)_{n \geq 0}$ denotes the sequence of Faber polynomials associated with $K$ and $\left(e_{n}\right)_{n \geq 0}$ will denote the canonical algebraic basis of $\mathbb{C}^{(\mathbb{N})}$.

Let $\mathcal{R} \subset \mathbb{C}^{\mathbb{N}}$ be a Banach space such that

(1) For any $k \in \mathbb{N}$, the map $\left(a_{n}\right)_{n \geq 0} \mapsto a_{k}$ is bounded.

(2) The set of finitely supported sequences $\mathbb{C}^{(\mathbb{N})}$ is dense in $\mathcal{R}$.

As $\mathbb{C}^{(\mathbb{N})}$ is dense in $\mathcal{R}$, each element $\varphi$ in $\mathcal{R}^{*}$ is characterized by the sequence $\left(\varphi_{n}\right)_{n \geq 0}$, where

$$
\varphi_{n}=\left\langle e_{n} ; \varphi\right\rangle_{\mathcal{R}}
$$

This allows us to identify $\mathcal{R}^{*}$ with a subset of $\mathbb{C}^{\mathbb{N}}$. Via this identification, we can translate the condition $(1)$ as " $\mathbb{C}^{(\mathbb{N})}$ is included in $\mathcal{R}^{*}$ ". 
Proposition 4.1. If $\varphi \in \mathcal{R}^{*}$ and $f \in \mathbb{C}^{(\mathbb{N})}$, the duality mapping is given by

$$
\langle f ; \varphi\rangle_{\mathcal{R}}=\sum_{n=0}^{\infty} f_{n} \varphi_{n} .
$$

If $f \in \mathcal{R}$ and $\varphi \in \mathbb{C}^{(\mathbb{N})} \subset \mathcal{R}^{*}$, this equality still holds.

Proof. Let $\varphi \in \mathcal{R}^{*}$ be finitely supported and let $n_{0}$ be such that $\left\langle e_{n} ; \varphi\right\rangle=0$ whenever $n \geq n_{0}$. From (2), we know that there exists a sequence $\left(g_{k}\right)_{k \geq 0}$ of elements in $\mathbb{C}^{(\mathbb{N})}$ such that

$$
g_{k} \underset{k \rightarrow \infty}{\longrightarrow} f \text { in } \mathcal{R} .
$$

To prevent any confusion, we will denote by $\left[g_{k}\right]_{n}$ the $n$-th element of the sequence $g_{k}$. Hence,

$$
\langle f ; \varphi\rangle_{\mathcal{R}}=\lim _{k \rightarrow \infty}\left\langle g_{k} ; \varphi\right\rangle_{\mathcal{R}}
$$

We also have $\left\langle g_{k} ; \varphi\right\rangle_{\mathcal{R}}=\sum_{n=0}^{n_{0}}\left[g_{k}\right]_{n} \varphi_{n}$. Therefore, using the hypothesis (1), we get that $\left[g_{k}\right]_{n} \underset{k \rightarrow \infty}{\longrightarrow} f_{n}$, for every $n$.

We obtain

$$
\langle f ; \varphi\rangle_{\mathcal{R}}=\lim _{k \rightarrow \infty}\left\langle g_{k} ; \varphi\right\rangle_{\mathcal{R}}=\sum_{n=0}^{\infty} f_{n} \varphi_{n} .
$$

The crucial condition needed for our Tauberian conditions is the following one.

(3) For any $\zeta \in \partial K$, the map $V_{\zeta}$, defined in $\mathbb{C}^{(\mathbb{N})} \subset \mathcal{R}^{*}$ by

$$
V_{\zeta}: \varphi \mapsto \sum_{n \geq 0} \varphi_{n} F_{n}(\zeta)
$$

is not bounded.

Theorem 4.2. Let $\mathcal{R} \subset \mathbb{C}^{\mathbb{N}}$ be a Banach space satisfying conditions (1) to (3) for $K$. Let $T \in B(\mathcal{X})$ be a weak type $\mathcal{R}$ operator with $\sigma(T) \subset K$. Then, in fact,

$$
\sigma(T) \subset \operatorname{int}(K) \text {. }
$$

Proof. Lemma 3.6 implies that

$$
C:=\sup \left\{\left\|\left(\left\langle F_{n}(T) x ; x^{*}\right\rangle\right)_{n \geq 0}\right\|_{\mathcal{R}} ;\left\|x^{*}\right\| \leq 1,\|x\| \leq 1\right\}<\infty .
$$

Using Proposition 4.1 we get, for every $\varphi \in \mathbb{C}^{(\mathbb{N})} \subset \mathcal{R}^{*}$,

$$
\begin{aligned}
\left\|\sum_{n \geq 0} \varphi_{n} F_{n}(T)\right\| & =\sup \left\{\left|\sum_{n \geq 0} \varphi_{n}\left\langle F_{n}(T) x ; x^{*}\right\rangle\right| ;\|x\| \leq 1,\left\|x^{*}\right\| \leq 1\right\} \\
& \leq \sup \left\{\left\|\left(\left\langle F_{n}(T) x ; x^{*}\right\rangle\right)_{n \geq 0}\right\|_{\mathcal{R}} ;\|x\| \leq 1,\left\|x^{*}\right\| \leq 1\right\}\|\varphi\|_{\mathcal{R}^{*}} \\
& \leq C\|\varphi\|_{\mathcal{R}^{*}} .
\end{aligned}
$$


Recall that, according to the spectral mapping theorem [5], we have $\sigma(P(A))=$ $P(\sigma(A))$ for every polynomial $P$. Hence if $\zeta \in \sigma(T)$, we have

$$
\left|\sum_{n \geq 0} \varphi_{n} F_{n}(\zeta)\right| \leq r\left(\sum_{n \geq 0} \varphi_{n} F_{n}(T)\right) \leq\left\|\sum_{n \geq 0} \varphi_{n} F_{n}(T)\right\| \leq C\|\varphi\|_{\mathcal{R}^{*}},
$$

where $r(T)=\sup \{|\lambda| ; \lambda \in \sigma(T)\}$ is the spectral radius of $T$. We deduce from this inequality that for any $\zeta \in \sigma(T)$, the map $V_{\zeta}$ is bounded in $\mathbb{C}^{(\mathbb{N})} \subset \mathcal{R}^{*}$.

This gives us

$$
\partial K \cap \sigma(T)=\varnothing
$$

Remark 4.3. A weaker version of Corollary 3.8 can be deduced from Theorem 4.2 (one has to suppose that $\sigma(T) \subset K$ ). One can notice that Corollary 3.9 cannot be deduced from Theorem 4.2.

A novelty of the approach of this section is that we can get a generalisation of the following result due to Mlak [11].

Theorem 4.4 ([11]). Let $\mathcal{H}$ be a Hilbert space and $T \in B(\mathcal{H})$. Suppose that $r(T) \leq 1$ and that for any $(x, y) \in \mathcal{H} \times \mathcal{H}$,

$$
z \mapsto\left\langle(z I-T)^{-1} x ; y\right\rangle \in H^{1}(\mathbb{D}) .
$$

Then $r(T)<1$.

To extend Mlak's result, we need a generalisation of Hardy spaces of the disc to more general domains. We follow the setting of [6].

Definition $4.5([6])$. For $p \in[1 ; \infty)$, a function $f$ analytic in $\mathbb{C} \backslash K$ is said to be of class $E^{p}(\mathbb{C} \backslash K)$ if there exists a sequence of rectifiable Jordan curves $C_{1}, C_{2}, \ldots$ in $\mathbb{C} \backslash K$ such that $C_{n}$ eventually surrounds each compact subset of $\mathbb{C} \backslash K$, and such that

$$
\sup _{n} \int_{C_{n}}|f(z)|^{p}|d z|<\infty .
$$

The following characterization ([6, Theorem 10.1]) of the functions in $E^{p}(\mathbb{C} \backslash K)$ is well adapted here as it uses the conformal representation $\phi$.

Theorem 4.6 ([6]). An analytic function $f$ is of class $E^{p}(\mathbb{C} \backslash K)$ if and only if,

$$
\sup _{r \in(1 ; 2)} \int_{|\phi(z)|=r}|f(z)|^{p}|d z|<\infty .
$$

We can now state the generalisation of Mlak's result [11] as a corollary to Theorem 4.2 .

Corollary 4.7. Suppose that $\lim _{n}\left|F_{n}(\zeta)\right|=0$ for no $\zeta \in \partial K$. Let $T \in B(\mathcal{X})$ be such that $\sigma(T) \subset K$.

If for any $x \in \mathcal{X}$ and $x^{*} \in \mathcal{X}^{*}$ we have

$$
z \mapsto\left\langle(z I-T)^{-1} x ; x^{*}\right\rangle \in E^{1}(\mathbb{C} \backslash K),
$$

then $\sigma(T) \subset \operatorname{int}(K)$. 
Remark 4.8. According to Remark 2.6, if $\partial K$ is an Alper curve with (possible) angles, the condition on the sequence $\left(\left|F_{n}(\zeta)\right|\right)_{n \geq 0}$ is automaticaly satisfied.

Lemma 4.9. Suppose that $\lim _{n}\left|F_{n}(\zeta)\right|=0$ for no $\zeta \in \partial K$. Then the space

$$
h_{p}:=\left\{\left(x_{n}\right)_{n \geq 0} ; f(z)=\sum_{n=0}^{\infty} x_{n} z^{n} \in H^{p}(\mathbb{D})\right\},
$$

with the norm

$$
\left\|\left(x_{n}\right)_{n \geq 0}\right\|=\left\|\sum_{n=0}^{\infty} x_{n} z^{n}\right\|_{H^{p}(\mathbb{D})}
$$

satisfies conditions (1) to (3).

Proof. It is easy to check that conditions (1) and (2) are satisfied. Let us prove that the condition (3) is also satisfied.

Suppose on the contrary that condition (3) does not hold. Then there exists $\zeta \in \partial K$ such that $V_{\zeta}$ is bounded in $\mathbb{C}^{(\mathbb{N})} \subset \mathcal{R}^{*}$. Let $a_{n}=F_{n}(\zeta)$ for every $n$.

According to the Hahn-Banach theorem, there exists a bounded extension of $V_{\zeta}$ to $\left(h^{p}\right)^{*}$ still denoted $V_{\zeta}$. Hence there exists $M \geq 0$ such that for every $x^{*}=\left(x_{n}^{*}\right)_{n \geq 0} \in\left(h^{p}\right)^{*}$,

$$
\left|V_{\zeta}\left(x^{*}\right)\right| \leq M\left\|\left(x^{*}\right)\right\|_{\left(h^{p}\right)^{*}}
$$

But $\|f(r .)\|_{H^{p}} \leq\|f\|_{H^{p}}$ for every $r \in(0 ; 1)$ and every $f \in H^{p}$. Thus, for every $\left(x_{n}^{*}\right)_{n \geq 0} \in\left(h^{p}\right)^{*}$,

$$
\begin{aligned}
\left\|\left(r^{n} x_{n}^{*}\right)_{n \geq 0}\right\|_{\left(h^{p}\right)^{*}} & =\sup \left\{\left|\left\langle\left(r^{n} x_{n}^{*}\right)_{n \geq 0} ;\left(x_{n}\right)_{n \geq 0}\right\rangle\right| ;\left\|\left(x_{n}\right)_{n \geq 0}\right\|_{h^{p}} \leq 1\right\} \\
& =\sup \left\{\left|\left\langle\left(x_{n}^{*}\right)_{n \geq 0} ;\left(r^{n} x_{n}\right)_{n \geq 0}\right\rangle\right| ;\left\|\left(x_{n}\right)_{n \geq 0}\right\|_{h^{p}} \leq 1\right\} \\
& \leq\left\|\left(x_{n}^{*}\right)_{n \geq 0}\right\|_{\left(h^{p}\right)^{*}} .
\end{aligned}
$$

So, for every $r \in(0 ; 1)$ and for every $\left(x_{n}^{*}\right)_{n \geq 0} \in\left(h^{p}\right)^{*}$,

$$
\left|\sum_{n=0}^{\infty} x_{n}^{*} r^{n} a_{n}\right| \leq M\left\|\left(x_{n}^{*}\right)_{n \geq 0}\right\|_{\left(h^{p}\right)^{*}} .
$$

Consequently, $\left\|\left(r^{n} a_{n}\right)_{n>0}\right\|_{h^{p}} \leq M$ for each $r \in(0 ; 1)$, and thus $\left(a_{n}\right)_{n>0}$ is in $h^{p}$. We have $f\left(e^{i \theta}\right)=\sum a_{n} e^{i n \theta} \in H^{p}(\mathbb{T})$ and $a_{n}=F_{n}(\zeta) \underset{n \rightarrow \infty}{\longrightarrow} 0$. This contradiction completes the proof.

Proof of Corollary 4.7. We get from Lemma 4.9 and Theorem 4.2 that it is sufficient to prove that for any $x \in \mathcal{X}$ and $x^{*} \in \mathcal{X}^{*}$ we have

$$
\left(\left\langle F_{n}(T) x ; x^{*}\right\rangle\right)_{n \geq 0} \in h^{1} .
$$


From Lemma 2.9 we get that for any $r>1$,

$$
\begin{aligned}
\int_{|\phi(z)|=r}\left|\left\langle(z I-T)^{-1} x ; x^{*}\right\rangle\right||d z| & =\int_{z \in r \mathbb{T}}\left|\left\langle(\psi(z) I-T)^{-1} x ; x^{*}\right\rangle\right|\left|\psi^{\prime}(z)\right||d z| \\
& =\int_{z \in r \mathbb{T}}\left|\sum_{n=0}^{\infty} z^{-n-1}\left\langle F_{n}(T) x ; x^{*}\right\rangle\right||d z| \\
& =\int_{z \in r^{-1} \mathbb{T}}\left|\sum_{n=0}^{\infty} z^{n}\left\langle F_{n}(T) x ; x^{*}\right\rangle\right| r|d z| .
\end{aligned}
$$

This proves that

$$
z \mapsto \sum_{n=0}^{\infty} z^{n}\left\langle F_{n}(T) x ; x^{*}\right\rangle \in H^{1}(\mathbb{D})
$$

Remark 4.10. The space $h^{1}$ is not an admissible sequence space, thus Corollary 4.7 cannot be deduced from Theorem 3.5.

\section{EXPlicit ESTIMATES}

5.1. General case. We suppose in this section that $\Gamma$ is an analytic Jordan curve. Let $\mathcal{E}$ be a Banach function space over $\mathbb{N}$ containing $\left(r^{n}\right)_{n \geq 0}$ for any $r \in(0 ; 1)$ and satisfying the condition

$$
\left\|\chi_{\{0, \ldots, n-1\}}\right\|_{\mathcal{E}} \underset{n \rightarrow \infty}{\longrightarrow} \infty .
$$

Let $T \in B(\mathcal{X})$ be a weak type $\mathcal{E}$ operator.

As we have supposed $\Gamma$ to be analytic, we get from Theorem 2.7 that the map $\psi: \overline{\mathbb{D}}^{c} \longrightarrow K^{c}$ has an analytic extension to ${\overline{r_{0}}}^{c}$ for some $r_{0}<1$.

Notation. For any $r \in\left(r_{0} ; 1\right)$, let $\Gamma_{r}$ be the analytic Jordan curve defined by

$$
\Gamma_{r}=\{\psi(z) ;|z|=r\}
$$

and let $K_{r}$ be the simply connected compact delimited by $\Gamma_{r}$.

We know from Theorem 3.5 that the spectrum of $T$ is included in $i n t(K)$. Having in mind possible applications in numerical analysis, it is the aim of this section to give an estimate of the "shrinking radius" $r \in\left(r_{0} ; 1\right)$ such $\sigma(T) \subset K_{r}$. In the case of the disc, this was done by N. Nikolski in [14]. Our estimate of the "shrinking radius" is given in terms of the constant $C(T, \mathcal{E})$ defined by

$$
C(T, \mathcal{E})=\sup \left\{\left\|\left(\left\langle F_{n}(T) x ; x^{*}\right\rangle\right)_{n \geq 0}\right\|_{\mathcal{E}} ;\left\|x^{*}\right\| \leq 1,\|x\| \leq 1\right\} .
$$

Notation. For $r \in\left(r_{0} ; 1\right)$ we denote

$$
p(r)=\inf _{r \leq|\phi(\zeta)|<1}\left\|\left(F_{n}(\zeta)\right)_{n \geq 0}\right\|_{\mathcal{E}}
$$


and, for $M>0$

$$
p^{-1}(M)= \begin{cases}r_{0} & \text { if }\left\{r \in\left(r_{0} ; 1\right) ; p(r) \leq M\right\}=\varnothing, \\ \sup \left\{r \in\left(r_{0} ; 1\right) ; p(r) \leq M\right\} & \text { otherwise. }\end{cases}
$$

Theorem 5.1. Let $T$ be a weak type $\mathcal{E}$ operator for the compact $K$ and set $r=p^{-1}(C(T, \mathcal{E}))$. Then

$$
\sigma(T) \subset K_{r} .
$$

Proof. We argue by contradiction. Suppose that $\sigma(T) \nsubseteq K_{r}$; then there exists $\lambda \in \partial \sigma(T) \backslash K_{r}$. From Lemma 3.7 we get that for any $\varepsilon>0$ and any $N \in \mathbb{N}$, there exist $x_{N} \in \mathcal{X}$ and $x_{N}^{*} \in \mathcal{X}^{*}$ satisfying $\left\|x_{N}\right\|=1,\left\|x_{N}^{*}\right\|=1$ and

$$
\left|\left\langle x_{N}^{*} ; F_{k}(T) x_{N}\right\rangle\right|>\left|F_{k}(\lambda)\right|-\varepsilon, \quad k \in\{0,1, \ldots N\} .
$$

By definition of $C(T, \mathcal{E})$, we have

$C(T, \mathcal{E}) \geq\left\|\left(\left\langle F_{n}(T) x_{N} ; x_{N}^{*}\right\rangle\right)_{n \geq 0}\right\|_{\mathcal{E}} \geq\left\|\left(F_{0}(\zeta), \ldots, F_{N}(\zeta), 0,0, \ldots\right)-\varepsilon \chi_{\{0, \ldots, N\}}\right\|_{\mathcal{E}}$.

Thus, for every $N \in \mathbb{N}$,

$$
\left\|\left(F_{0}(\lambda), F_{1}(\lambda), \ldots, F_{N}(\lambda), 0,0, \ldots\right)\right\|_{\mathcal{E}} \leq C(T, \mathcal{E}) .
$$

But $F_{n}(\lambda) \sim \phi(\lambda)^{n}, n \rightarrow \infty$, and $|\phi(\lambda)|<1$. Therefore $\left(F_{n}(\lambda)\right)_{n \geq 0} \in \mathcal{E}$ and

$$
\left\|\left(F_{n}(\lambda)\right)_{n \geq 0}\right\|_{\mathcal{E}} \leq C(T, \mathcal{E}) .
$$

Using $\lambda \notin K_{r}$, and taking into account the definition of $r$ as $r=p^{-1}(C(T, \mathcal{E}))$, we have

$$
\left\|\left(F_{n}(\lambda)\right)_{n \geq 0}\right\|_{\mathcal{E}}>C(T, \mathcal{E}) .
$$

This contradiction completes the proof.

5.2. Concrete examples. In case of some concrete compacts, the previously described quantities can be explicitly estimated. We begin with the case of a compact $K$ such that $K_{r_{0}}$ is convex. The following proof has been kindly suggested to the author by a referee.

Corollary 5.2. Suppose $r<1$ and $1 \leq p<\infty$. Let $T$ be a weak type $l^{p}$ operator for a compact $K$ delimited by an analytic Jordan curve. Suppose that $K_{r_{0}}$ is convex and

Then

$$
C\left(T, l^{p}\right) \leq\left(\frac{1}{1-r^{p}}\right)^{1 / p}-\left(\frac{1}{1-r_{0}^{p}}\right)^{1 / p}
$$

$$
\sigma(T) \subset K_{r}
$$

Proof. One can get from the inequality of Kövari and Pommerenke [10] (cf. Theorem 2.8) and a change of variable that for any $z \in \Gamma_{r_{0}}$ and any $n \in \mathbb{N}$, we have

$$
\left|F_{n}(z)-\phi(z)^{n}\right| \leq r_{0}^{n}
$$


As $F_{n}-\phi^{n}$ is an analytic function on $\overline{\mathbb{C}} \backslash K_{0}$ and vanishes at $\infty$, the maximum principle gives us that for any $z \notin K_{r_{0}}$,

$$
\left|F_{n}(z)-\phi(z)^{n}\right| \leq r_{0}^{n} .
$$

Let $w \in \mathbb{C}$ be such that $|w|>r_{0}$. Then

$$
\begin{aligned}
\left\|\left(F_{n}(\psi(w))\right)_{n \geq 0}\right\|_{l^{p}} & =\left\|\left(w^{n}+\left(F_{n}(\psi(w))-w^{n}\right)\right)_{n \geq 0}\right\|_{l^{p}} \\
& \geq\left\|\left(w^{n}\right)_{n \geq 0}\right\|_{l^{p}}-\left\|\left(r_{0}^{n}\right)_{n \geq 0}\right\|_{l^{p}} .
\end{aligned}
$$

Thus, for every $t \in\left(r_{0} ; 1\right)$, we have

$$
\begin{aligned}
p(t) & \geq\left(\sum_{n=0}^{\infty} t^{n p}\right)^{1 / p}-\left(\sum_{n=0}^{\infty}\left(r_{0}\right)^{n p}\right)^{1 / p} \\
& \geq\left(\frac{1}{1-t^{p}}\right)^{1 / p}-\left(\frac{1}{1-\left(r_{0}\right)^{p}}\right)^{1 / p} .
\end{aligned}
$$

The conclusion follows now from Theorem 5.1.

We now turn to the more specific case of an ellipse. Let $K$ be the compact delimited by the ellipse with the foci -1 and 1 , and semi-axes

$$
a=\frac{1}{2}\left(R+\frac{1}{R}\right) \quad \text { and } \quad b=\frac{1}{2}\left(R-\frac{1}{R}\right),
$$

with $R>1$. Then (cf. Section 2) the function $\psi$ is given by

$$
\psi(w)=\frac{1}{2}\left(R w+\frac{1}{R w}\right)
$$

and the sequence of Faber polynomials is given by

$$
F_{n}(z)=\frac{2}{R^{n}} C_{n}(z), \quad n \geq 1
$$

where $\left(C_{n}\right)_{n \in \mathbb{N}}$ is the sequence of Chebychev polynomials. We also have

$$
F_{n}(\psi(w))=w^{n}+\frac{1}{R^{2 n} w^{n}} \quad(n \geq 1) .
$$

Corollary 5.3. Let $K$ be the elliptic compact set described above. Suppose $r<1$ and $1 \leq p<\infty$. Let $T \in B(\mathcal{X})$ be a weak type $l^{p}$ operator for $K$. Suppose that

$$
C\left(T, l^{p}\right) \leq\left(\frac{1}{1-r^{p}}\right)^{1 / p}-\left(\frac{1}{1-\left(\frac{1}{r R^{2}}\right)^{p}}\right)^{1 / p}
$$

Then

$$
\sigma(T) \subset K_{r}
$$

Remark 5.4. This estimate is better than the one given in Corollary 5.2 as $r_{0}=1 / R>1 / r R^{2}$ for $r>r_{0}$. 
Proof. It is easy to verify that $r_{0}=1 / R$. Let $w \in \mathbb{C}$ be such that $|w|>1 / R$. Then

$$
\begin{aligned}
\left\|\left(F_{n}(\psi(w))\right)_{n \geq 0}\right\|_{l^{p}} & =\left\|\left(w^{n}+\frac{1}{R^{2 n} w^{n}}\right)_{n \geq 0}\right\|_{l^{p}} \\
& \geq\left\|\left(w^{n}\right)_{n \geq 0}\right\|_{l^{p}}-\left\|\left(\frac{1}{R^{2 n} w^{n}}\right)_{n \geq 0}\right\|_{l^{p}} .
\end{aligned}
$$

Thus, for every $t \in(1 / R ; 1)$,

$$
\begin{aligned}
p(t) & \geq\left(\sum_{n=0}^{\infty} t^{n p}\right)^{1 / p}-\left(\sum_{n=0}^{\infty}\left(\frac{1}{t R^{2}}\right)^{n p}\right)^{1 / p} \\
& \geq\left(\frac{1}{1-t^{p}}\right)^{1 / p}-\left(\frac{1}{1-\left(\frac{1}{t R^{2}}\right)^{p}}\right)^{1 / p} .
\end{aligned}
$$

The conclusion follows now from Theorem 5.1.

Acknowledgement: I would like to express my sincere gratitude to my advisor Catalin Badea for proposing the topic of this paper and for his guidance and support.

I would also like to thank the two referees for their constructive remarks, critical reading and suggestions for improvements.

\section{REFERENCES}

[1] A. Atzmon; A. Eremenko; M. Sodin, Spectral inclusion and analytic continuation. Bull. London Math. Soc. 31 (1999), no. 6, 722-728.

[2] C. Badea, S. Grivaux, Faber-hypercyclic operators. Israel J. Math. 165 (2008), 43-65.

[3] C. Badea, S. Grivaux, Size of the peripheral point spectrum under power or resolvent growth conditions. J. Funct. Anal. 246 (2007), no. 2, 302-329.

[4] B. Beckermann, Image numérique, GMRES et polynômes de Faber. C. R. Math. Acad. Sci. Paris 340 (2005), no. 11, 855-860.

[5] J. Conway, A course in functional analysis. Second edition. Graduate Texts in Mathematics, 96. Springer-Verlag, New York, 1990. xvi+399 pp. ISBN: 0-387-97245-5.

[6] P. Duren, Theory of $H^{p}$ spaces. Pure and Applied Mathematics, Vol. 38 Academic Press, New York-London 1970 xii +258 pp.

[7] M. Eiermann; R. Varga, Zeros and local extreme points of Faber polynomials associated with hypocycloidal domains. Electron. Trans. Numer. Anal. 1 (1993), Sept., 49-71.

[8] D. Gaier, Lectures on complex approximation. Translated from the German by Renate McLaughlin. Birkhäuser Boston, Inc., Boston, MA, 1987. xvi+196 pp. ISBN: 0-8176$3147-\mathrm{X}$

[9] M. X. He ; E. B. Saff, The zeros of Faber polynomials for an m-cusped hypocycloid. J. Approx. Theory 78 (1994), no. 3, 410-432.

[10] T. Kövari, Pommerenke, On Faber polynomials and Faber expansions. Math. Zeitschrift 99 (1967) 193-206.

[11] W. Mlak, On a theorem of Lebow. Ann. Polon. Math. 35 (1977/78), no. 1, 107-109.

[12] O. Nevanlinna, Convergence of iterations for linear equations. Lectures in Mathematics ETH Zürich. Birkhäuser Verlag, Basel, 1993. viii+177 pp. ISBN: 3-7643-2865-7 
[13] N. Nikolski, A Tauberian theorem for the spectral radius. Sibirsk. Mat. Zh. 18 (1977), no. 6, 1367-1372; English transl., Siberian Math. J. 18 (1977), no. 6, 969-972 (1978).

[14] N. Nikolski, Estimates of the spectral radius and the semigroup growth bound in terms of the resolvent and weak asymptotics. Algebra i Analiz 14 (2002), no. 4, 141-157; translation in St. Petersburg Math. J. 14 (2003), no. 4, 641-653

[15] I. E. Pritsker, On the local asymptotics of Faber polynomials. Proc. Amer. Math. Soc. 127 (1999), no. 10, 2953-2960.

[16] P. K. Suetin, Series of Faber polynomials. Translated from the 1984 Russian original by E. V. Pankratiev [E. V. Pankrat'ev]. Analytical Methods and Special Functions, 1. Gordon and Breach Science Publishers, Amsterdam, 1998. xx+301 pp. ISBN: 90-5699-058-6

[17] K-C. Toh; L. Trefethen The Kreiss matrix theorem on a general complex domain. SIAM J. Matrix Anal. Appl. 21 (1999), no. 1, 145-165.

[18] J. M. A. M. van Neerven, Exponential stability of operators and operator semigroups. J. Funct. Anal. 130 (1995), no. 2, 293-309.

[19] G. Weiss, Weakly $l^{p}$-stable linear operators are power stable. Internat. J. Systems Sci. 20 (1989), no. 11, 2323-2328.

Laboratoire Paul Painlevé, UMR CNRS 8524, BÂt. M2, Université Lille 1, 59655

Villeneuve D'Ascq Cedex, France

E-mail address: oscar.devys@math.univ-lille1.fr ; oscar.devys@gmail.com 